\title{
Outflows from Accretion Disks around Compact Objects
}

\author{
Cheng-Liang $\mathrm{Jiao}^{1}$ and $\mathrm{Xue-Bing} \mathrm{Wu}^{2}$ \\ ${ }^{1}$ Radio Astronomy Research Center, Korea Astronomy and Space Science Institute, \\ 776 Daedeokdae-ro, Yuseong-gu, Daejeon, Republic of Korea \\ email: chengliang.jiao@gmail.com \\ ${ }^{2}$ Dept. of Astronomy, Peking University, \\ Beijing 100871, China \\ email: wuxb@pku.edu.cn
}

\begin{abstract}
We solve the set of hydrodynamic equations for accretion disks in the spherical coordinates $(r \theta \phi)$ to obtain the explicit structure along the $\theta$ direction. The results display thinner, quasi-Keplerian disks for Shakura-Sunyaev Disks (SSDs) and thicker, sub-Keplerian disks for Advection Dominated Accretion Flows (ADAFs) and slim disks, which are consistent with previous popular analytical models, while an inflow region and an outflow region always exist, which supports the results of some recent numerical simulation works. Our results indicate that the outflows should be common in various accretion disks and stronger in slim disks and ADAFs.
\end{abstract}

Keywords. accretion, accretion disks - outflow - hydrodynamics - black hole physics

\section{Introduction}

Recent development in observations has shown more and more evidence of outflows in accretion systems, such as Sgr A* (Marrone et al. 2006; Xie \& Yuan 2008), soft Xray transients (Loeb et al. 2001) and quasars with blueshifted absorption lines (e.g., PG1115+80; Chartas et al. 2003). Many two- or three-dimensional numerical simulations of accretion disks have also found outflow in their results (e.g. Stone et al., 1999; Igumenshchev \& Abramowicz, 2000; Okuda et al. 2005; Ohsuga et al.2005; Ohsuga \& Mineshige 2007; Ohsuga et al. 2009; Yuan et al. 2012; Narayan et al. 2012). On the other hand, most accretion disk models in literature do not contain outflows. This arises from the fact that in these models, many simplifications are applied in the vertical direction, with vertical velocity set to zero and the vertical variation of velocity field neglected. One need to waive these assumptions to study outflows. Analytical works in this field are still quite limited, among which are contributions from Narayan \& Yi (1995), Xu \& Chen (1997), Blandford \& Begelman (1999, 2004), Xue \& Wang (2005), Sadowski et al. (2010), etc. (see Jiao \& Wu 2011 for a detailed discussion of the contributions and caveats of these works). As analytic disk models are still the only accessible way of making direct link between theory and observations, e.g. fitting the spectra of accretion-powered astrophysical systems, it is very important to improve the study in this field.

\section{Our Method}

We consider an accretion flow around a compact object such as a black hole, and the self-gravitational effects are ignored. The flow is assumed to be steady and axisymmetric. We use the Newtonian gravitational potential and alpha prescription of viscosity. The 
equation set consists of the equations of continuity, motion and energy, which are written in spherical coordinates $(r, \theta, \phi)$. We use the advective factor $f \equiv Q_{\mathrm{adv}} / Q_{\mathrm{vis}}$ for the energy equation, which means that a fraction $f$ of the dissipated energy is advected as stored entropy and a fraction $(1-f)$ is lost due to radiation, and $f$ is assumed to be a constant in our calculation.

We adopt the self-similar assumptions in the radial direction (Narayan \& Yi 1995, Xue \& Wang 2005). Then the partial differential equations are changed to ordinary differential equations (ODEs) in $\theta$-direction with four input parameters $\left(\alpha, f, \gamma_{\text {equ }}, n\right)$, in which $\gamma_{\text {equ }}$ is determined by the heat capacity ratio $\gamma$ and gas pressure ratio $\beta$, and $n(\equiv-\partial \ln \rho / \partial \ln r)$ describes how the density changes along radius. This set of ODEs can be numerically solved with symmetric boundary conditions to the equatorial plane. The density on the equatorial plane is set to be 1, which is normalized by a scale factor relative to the accretion rate. The improvement in this model is that the existance and structure of outflow is obtained by calculation, rather than assumption. No arbitrary boundary conditions are introduced in our calculation.

\section{Results}

The velocity fields of four typical solutions of our model are shown in Figure 1. The accretion flow contains an inflow region near the equatorial plane and an outflow region above the inflow region in general. The calculation starts from the equatorial plane, and both the density and pressure get close to zero at certain inclinations, at which we stop the calculation and regard that as the upper boundary. We suspect that the region above the upper boundary contains outflow which does not obey the self-similar radial distribution.

(a)

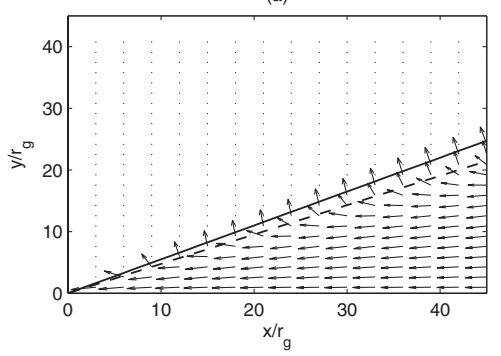

(c)

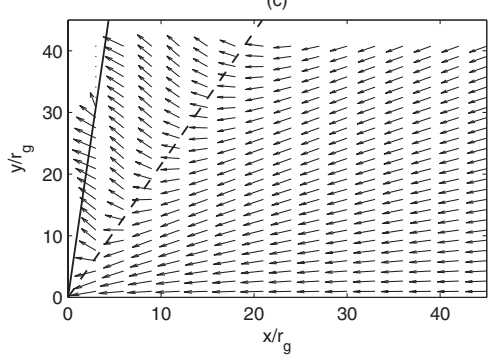

(b)

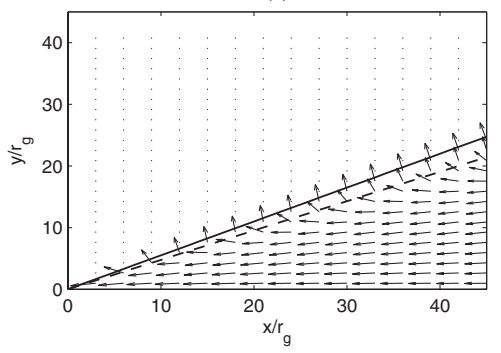

(d)

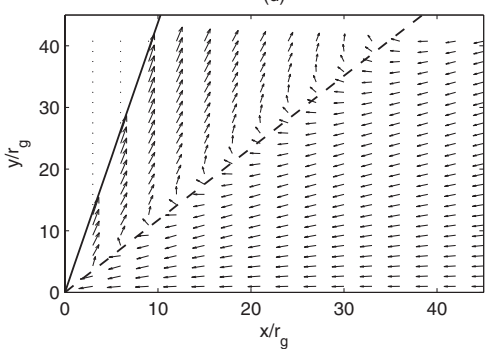

Figure 1. The velocity fields of four typical solutions. Figures (a) and (b) correspond to $f=0.01$, while (c) and (d) correspond to $f=1$. Figures (a) and (c) are gas-pressure dominated, while (b) and (d) are radiation-pressure dominated. The lengths of arrows indicate the absolute values of the vector $\overrightarrow{v_{r}}(\theta)+\overrightarrow{v_{\theta}}(\theta)$, which are scaled logarithmically. The solid lines indicate the upper boundary of the accretion flows, while the dashed lines indicate the boundary between inflow and outflow. 
It can also be seen from Figure 1 that advection-dominated accretions tend to have much thicker disk and much stronger outflow, while accretion flows with little advection have much thinner disk and only negligible outflow. If we set the advective factor $f$ to be even smaller, e.g. $10^{-6}$, then the structure of the disk will be nearly identical to the assumptions used in the famous Shakura-Sunyaev disk (Shakura \& Sunyaev 1973), i.e. very thin disk, Keplerian rotation, etc., as shown in Figure 2. It should be noted that we can not set $f=0$ as it will make the equations have no solutions.

The solution dependance on the input parameters are also studied. It is worth noting that the accretion flow does not need to be advection-dominated to have a non-negligible outflow region. Actually for $f>0.1$, there is already a considerable outflow region, though the mass outflow rate still requires a large $f$ to be significant, due to the fact that density is higher near the equatorial plane. When the disk is radiation-pressure dominated, the mass outflow rate will become larger as the advetive factor $f$ increases, reaching a comparable level as the mass inflow rate when $f$ is close to 1 . In this sense, slim disks, in which both advection and radiation pressure are dominant, are likely to have the strongest outflow in the normalized measurement.

Parameter $n$ describes how the density varies in the radial direction and is set as an input parameter in our calculation. Ideally, it should be determined by physics, which we will investigate in future work. It is worth noting that for a large range of $n$, the structure of accretion flow does not change much, except for $n$ close to 1.5 , which is a critical value at which the inflow/outflow pattern disappears and all the streamlines become straight lines directly pointing at the central accretor. This is the case studied in Narayan \& Yi 1995, and it can be reproduced in our calculation.
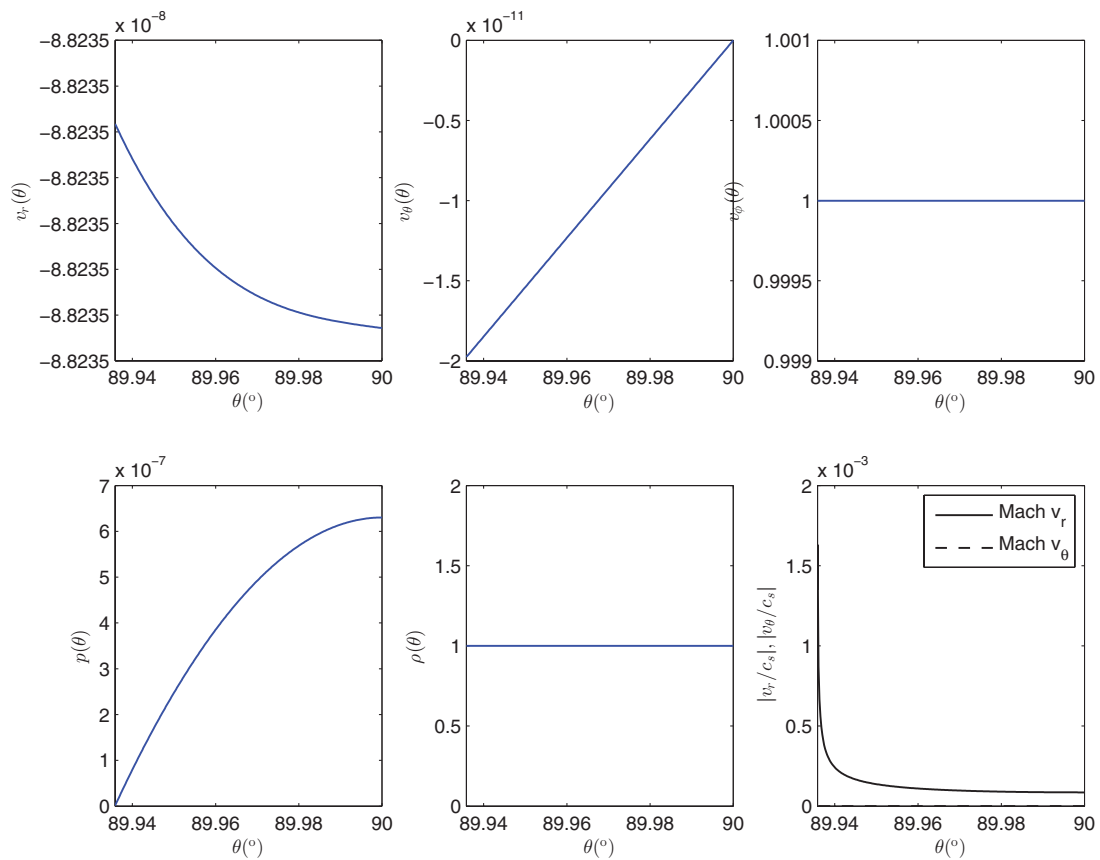

Figure 2. The solution corresponding to the SSD model. Here $\alpha=0.1, n=1.3, f=10^{-6}$ and $\gamma_{\text {equ }}=5 / 3$, which correspond to gas pressure dominated monatomic ideal gas. 


\section{Discussions and Conclusions}

We calculate the structure of accretion flows along the $\theta$-direction and find that outflows are common in all kinds of accretion modes. The accretion flow generally consists of three regions: an inflow region near the equatorial plane, and outflow region above the inflow region, and a third region close to the polar axis which does not obey self-similarity and may also consist of outflow.

Advection-dominated accretion flows tend to have strong outflows, while accretion flows with little advection only have very weak outflow. Our model can also reproduce the assumptions of SSDs when the advection is set to extremely low. As SSDs have both secular and thermal instability when $\beta<2 / 5$, and our model essentially descends to a SSD when $f$ is extremely small, our model could potentially be unstable in some parameter set and stable in others.

There are still caveats in our model, e.g. the $\alpha p$ prescription of viscosity, Newtonian potential, constant advective factor, constant gas pressure ratio, neglect of convection and magnetic fields, self-similar assumptions, etc. These are all directions of improvement in the future.

\section{References}

Abramowicz, M. A., Chen, X., Kato, S., Lasota, J.-P., \& Regev, O. 1995, ApJ, 438, L37

Blandford, R. D. \& Begelman, M. C. 1999, MNRAS, 303, L1

Blandford, R. D. \& Begelman, M. C. 2004, MNRAS, 349, 68

Chartas, G., Brandt, W. N., \& Gallagher, S. C. 2003, ApJ, 595, 85

Igumenshchev, I. V. \& Abramowicz, M. A. 2000, ApJS, 130, 463

Jiao, C.-L. \& Wu, X.-B. 2011, ApJ, 733, 112

Loeb, A., Narayan, R., \& Raymond, J. C. 2001, ApJ, 547, L151

Marrone, D. P., Moran, J. M., Zhao, J.-H., \& Rao, R. 2006, ApJ, 640, 308

Narayan, R., Sadowski, A., Penna, R. F., \& Kulkarni, A. K. 2012, arXiv:1206.1213

Narayan, R. \& Yi, I. 1995, ApJ, 444, 231

Ohsuga, K. \& Mineshige, S. 2007, ApJ, 670, 1283

Ohsuga, K., Mineshige, S., Mori, M., \& Kato, Y. 2009, PASJ, 61, L7

Ohsuga, K., Mori, M., Nakamoto, T., \& Mineshige, S. 2005, ApJ, 628, 368

Okuda, T., Teresi, V., Toscano, E., \& Molteni, D. 2005, MNRAS, 357, 295

Sạdowski, A., Abramowicz, M., Bursa, M., et al. 2011, A\&\&A, 527, A17

Shakura, N. I. \& Sunyaev, R. A. 1973, A\& A, 24, 337

Stone, J. M., Pringle, J. E., \& Begelman, M. C. 1999, MNRAS, 310, 1002

Xie, F.-G. \& Yuan, F. 2008, ApJ, 681, 499

Xu, G. \& Chen, X. 1997, ApJ, 489, L29

Xue, L. \& Wang, J. 2005, ApJ, 623, 372

Yuan, F., Bu, D., \& Wu, M. 2012, arXiv:1206.4173 